\title{
Attending to women's sexual health in Bahrain: does physician's gender make a difference?
}

\author{
Z. Al-Mohsen, ${ }^{1}$ N. Grant, ${ }^{2}$ M.A. Obaidat, ${ }^{2}$ H. Al-Farra, ${ }^{1}$ N. Budhaish ${ }^{7}$ and W. Al-Farra
}

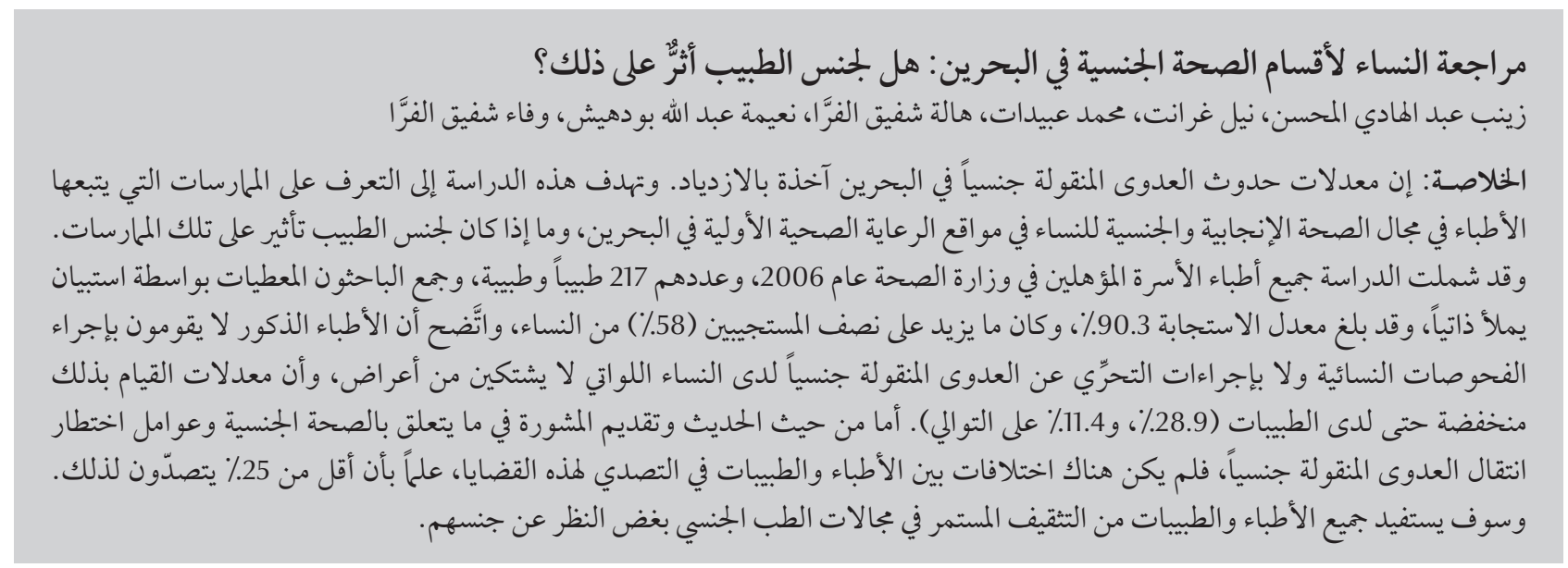

ABSTRACT There is a rising incidence of sexually transmitted infections (STIs) in Bahrain. This study aimed to determine physician practices with regard to sexual and reproductive health in women in the primary care setting in Bahrain, and to ascertain if physician gender affected these. The study included all eligible Ministry of Health family physicians (217) in 2006 and data were collected by a self-completed questionnaire; the response rate was $90.3 \%$. Over half (58\%) of the responding physicians were female. Male physicians did not undertake gynaecological examinations nor carry out STI screening procedures for asymptomatic women, and rates for women physicians were low ( $28.9 \%$ and $11.4 \%$ respectively). As regards to identification of and counselling for sexual health and STI risk factors, there were no differences between male and female physicians in addressing these issues with less than $25 \%$ doing so. All physicians would benefit from continuing education in the area of sexual medicine regardless of their gender.

Prise en charge de la santé génésique et sexuelle des femmes à Bahreïn : consulter un médecin homme ou un médecin femme fait-il une différence?

RÉSUMÉ L'incidence des infections sexuellement transmissibles est en augmentation à Bahreïn. La présente étude visait à déterminer les pratiques des médecins dans le domaine de la santé génésique et sexuelle des femmes en établissements de soins de santé primaires à Bahreïn, et cherchait à savoir si le fait que le médecin était un homme ou une femme faisait une différence. L'étude a inclus tous les médecins de famille éligibles travaillant dans des établissements dépendant du ministère de la Santé (217) en 2006. Les données ont été recueillies par auto-questionnaire. Le taux de réponse était de 90,3\%. Plus de la moitié (58\%) des médecins ayant répondu était des femmes. Si les médecins hommes ne réalisaient pas d'examen gynécologique ni de dépistage d'infections sexuellement transmissibles chez les femmes asymptomatiques, les médecins femmes, quant à elles, étaient peu nombreuses à les réaliser (28,9\% et $11,4 \%$ respectivement). Aucune différence n'a été observée entre la pratique des médecins hommes et des médecins femmes concernant l'identification des facteurs de risque pour les infections sexuellement transmissibles et l'offre de conseils pour la santé sexuelle. En effet, ils étaient moins de $25 \%$ à aborder ces questions avec leurs patientes. Tous les médecins, homme ou femme, tireraient avantage d'une formation continue dans le domaine de la médecine sexuelle. 


\section{Introduction}

The World Health Organization (WHO) estimates that 2.7 million new cases of HIV occurred throughout the world in 2008 in men and women aged 15-49 years, and in the Middle East, the estimated prevalence of $\mathrm{HIV}$ is $0.2 \%$ [1].

A rising incidence of sexually transmitted infections (STIs) in Bahrain is giving cause for concern. From 2005 to 2008, reported cases of gonorrhoea increased from 212 to 419 [2]. In the same year (2008), the total number of STIs reported was 918 and underreporting has been identified as an issue. In one recent study, only $28 \%$ of STIs diagnosed by physicians were reported [Al-Dhubaib et al., unpublished audit, 2007]. Part of this problem may lie in the complexity of the reporting system used by the Ministry of Health (MOH). Physicians are required to complete a specific notification form and send it to the nursing section in the health centre for further action; some physicians choose to do so by asking the patient to deliver the form, whereas others complete the task personally. There is a risk that the former approach may result in data loss due to patient confusion, worry about confidentiality or mere lack of time. In short, the reporting system may compound physician reluctance to address sexual health and infection.

Women and men are equally vulnerable to STIs which can have adverse impacts on psychological, physical and reproductive health. STIs, and especially HIV, carry a social stigma, making them a sensitive issue to address for both physician and patient, and this dynamic can be complicated further by physician gender.

In Bahrain, where possible, women patients are cared for by women physicians who conduct all clinics related to women's reproductive health including ante- and postnatal care, and well-woman assessments. Male physicians, in general, care for women in non-elective situations, for example, in emergencies and for episodic care.

Although many patients initially attend primary care services for STIs [3], and primary care is an important and attractive setting for the delivery of sexual and reproductive health care (with the potential for STI control), many primary care physicians feel that they do not have the necessary training and experience to respond to these demands [4].

The aim of this study was to assess physician practices with regard to issues of sexual and reproductive health in women in the primary care setting in Bahrain, and to ascertain what role was played by physician gender.

\section{Methods}

A descriptive, cross-sectional survey was carried out in July 2006, in all 21 Ministry of Health primary health care centres in Bahrain, and involved all practising primary care physicians.

A questionnaire, adapted from a previous study and with the permission of the author [5], addressed demographic data and STI-related practice behaviour of those physicians who routinely encountered women patients in their clinical practice.

The self-completed questionnaire in English consisted of 7 statements and participants were asked to respond using a 5-point scale that ranged from "none of the time" to "all of the time". when appropriate The statements were as follows:

- I personally perform routine gynaecological examinations on my patients.

- I test asymptomatic sexually active females in my practice for gonorrhoea.

- I counsel my patients about STIs/ HIV transmission and prevention.

- I ask my patients about sexual activity.

- I ask my patients about the use of condoms.
- I ask my patients about the number of past sexual partners.

- I ask my patients about past history of STIs.

A pilot study was carried out on 10 family practice residency programme physicians to assess the questionnaire and the practicality of completing it in the clinic setting. Following this, the questionnaires were personally distributed to and collected from all physicians eligible for inclusion in the study. All primary care physicians working during morning or afternoon shifts and registered with the $\mathrm{MOH}$ in 2006 were eligible for inclusion. Physicians who were not currently practising (being in administrative positions), those working as tutors in family practice programmes and those on leave were excluded.

Questions 1 and 2 involved clinical examination as opposed to communication behaviour and so were separated from the rest of the items for analysis. Responses were aggregated into "all of the time when appropriate" and "less than all of the time" to highlight consistency of behaviour.

\section{Ethical considerations}

During the planning phase of the research, permission was sought from the Bahrain Ministry of Health ethical committee. Support for the study was sought and given by the chairmen of the health centres involved.

Participants were given a clear written account attached to the questionnaire of the nature of the study, measures to be taken to protect confidentiality, and the benefit and value of the study, and their informed verbal consent was requested.

\section{Data analysis}

SPSS, version 14.0 was used for data analysis. Counts and percentages were calculated for personal data, and chisquared tests were applied to all items concerning practice behaviour. 


\section{Results}

Out of a total of 250 physicians, 33 were excluded from the study because of their non-involvement in clinical work or absence on leave; this was ascertained by the official listing of physicians provided by MOH. Thus 217 eligible physicians received a copy of the questionnaire and 196 were returned, a response rate of $90.3 \%$. The response rate among males and females was $94.3 \%$ and $87.7 \%$ respectively. Of the 21 eligible physicians excluded, 4 declined to participate at the outset and 17 did not complete the questionnaire due to lack of time or losing it. There was no follow-up of physicians who elected not to participate in the study.

\section{Respondents' characteristics}

As regards sex and age, $58 \%$ of respondents were female and $72.8 \%$ were over 35 years. Around $70 \%$ were graduates of the family medicine residency programme and the remainder were general practitioners, and $75 \%$ had been in primary care practice for more than 5 years.

\section{Physician practices}

With regard to the first item in the questionnaire, none of the male physicians undertook gynaecological examinations and few of the female physicians performed gynaecological examination and gonorrhoea screening (Table 1). Only 1 male physician answered positively to testing asymptomatic sexually active females for gonorrhoea.

No significant differences were found between male and female physicians with regard to STI counselling, and enquiring about history of sexual activity, condom use, sexual partners or past STIs (Table 2). Male physicians were less comfortable talking about sexual activity issues with their female patients. The Pearson chi-squared tests yielded no significant findings in any of the items.

\section{Discussion}

We found that no gynaecological examinations were carried out by male physicians, in keeping with local cultural norms. With regard to screening for gonorrhoea, only one male physician answered positively. It can be reasonably inferred that this did not include gynaecological examination. Rates for screening for gonorrhoea by female physicians, however, were low and much lower than rates for gynaecological examination ( $11.4 \%$ versus $28.9 \%$ ). The reasons for this discrepancy are not clear and require further study.

As regards communication practices relevant to the identification of risks for STIs, preventive measures and sexual history, no significant differences were found that could be attributable to physician's gender. These results are in contrast to other studies' findings, where female gender has been shown to be an important positive factor in the provision of effective STI care and prevention for women [6-9].

In addition, in regions where the cultural norms are different from Bahrain, studies have shown that women patients have a preference for same gender care, which in turn delivers enhanced care [10]. In Bahrain despite the fact that women receive care from female physicians, equivalent "care benefits" are not being realized [Al-Alawi, unpublished research, 2003], and rates of desirable behaviours on the part of women physicians were low in comparison with other studies [11]. The reasons for this were not explored in our study, but the result was surprising given that women physicians have more opportunity to ask their female patients about sexual health issues, as they conduct all clinics pertaining to women's reproductive health.

Clinical practice guidelines regarding sexual health and disease, although not emphasized in antenatal and postnatal protocols, are present in Bahrain. However, it appears that the opportunity to address sexual health issues is not taken. Despite a local residency programme with emphasis on communication and sexual history taking skills, residency training was found not to convey any advantage in rates of desirable practice behaviours. In contrast, other studies, albeit carried out in different cultures, have shown that training in communication skills can have a positive influence on sexual history comfort levels [12].

A number of factors may be responsible for the low level of effective sexual health care behaviour. For example, there may be an over-riding problem in the area of knowledge and skills, as has been demonstrated in research studies carried out in countries as diverse as Pakistan and the United States of America (USA), as well as Bahrain

\begin{tabular}{|c|c|c|c|c|}
\hline \multirow[t]{3}{*}{ Gender } & \multicolumn{2}{|c|}{ Performing gynaecological examinations } & \multicolumn{2}{|c|}{ Screening for gonorrhoea } \\
\hline & Less than all the time & All of the time & Less than all the time & All of the time \\
\hline & No. (\%) & No. (\%) & No. $(\%)$ & No. (\%) \\
\hline Male $(n=82)$ & $82(100.0)$ & $0(0.0)$ & $81(98.8)$ & $1(1.2)$ \\
\hline Female $(n=114)$ & $81(71.1)$ & $33(28.9)$ & $101(88.6)$ & $13(11.4)$ \\
\hline Total & $163(83.2)$ & 33 (16.8) & 182 (92.9) & $14(7.1)$ \\
\hline
\end{tabular}


[5,13, Al-Alawi, unpublished research, 2003]. In a study of 1600 physicians in the USA, Wiesenfeld found inadequacies in knowledge of treatment protocols and screening practices resulting in compromised care for women [5].

Further, the lack of emphasis on STI-related clinical practice guidelines for the more formal components of care, such as ante and postnatal care and well-woman examinations, may represent one aspect of care that needs to be addressed by policy-makers.

In Bahrain, in a study of physicians' perceptions of factors inhibiting discussion of sexual health issues with patients, Al-Alawi et al. reported that, in addition to knowledge and skill gaps in terms of human sexuality, limited consultation time, lack of privacy, language barriers and physician embarrassment were the main factors (Al-Alawi, unpublished research, 2003).

The first three of these problems (time, privacy and language) together with the somewhat confusing reporting system in the health centre which might result in negative feedback, are, at first glance, logistical but may veil a more fundamental type of problem. Many problems in the primary care setting are of a psychosocial nature, and time is needed to address them [14]. The need to acknowledge this fact and accommodate its implications is a matter for health care policy-makers to address.

The embarrassment factor is more complex. Its roots may lie in the culture, in ethics, e.g. conflict between individual rights and public health policy, or in an innate modesty and/or shame on the part of physicians and patients, and in physicians' perceptions of their patients' sensitivities (Al-Alawi, unpublished research, 2003). Physician gender as a cause of this reluctance is not well substantiated. For example, Aschka et al., in their study of the management by male physicians of sexual problems in male patients in family practice, found that physician reluctance to address sexual health issues exceeded that of their patients, a finding confirmed by the results that revealed that $84 \%$ of male patients considered it important to talk with their family physician about their sexual concerns [15]. More than two-thirds of the patients would have liked their physicians to signal their open-mindedness by directly addressing sexual topics during the consultation [15]. Qualitative research initiatives could shed light on both the roots of physicians' underlying discomfort as well as the expectations of patients, and provide some understanding of the inter-relationships of these interacting dimensions.

The primary health care setting provides an opportunity to attend to clients' sexual health. Given the still relatively low prevalence of HIV and AIDS in most countries in the Eastern Mediterranean region, utilizing this opportunity could help to prevent the spread of HIV/AIDS reaching epidemic status, when these diseases spread beyond the high risk groups [16].

There are some limitations to the study. The responses of the physicians were self-reported and may not correlate with their actual practice. In addition, some physicians may have been less likely to return the questionnaire as it may indicate poor performance.

In conclusion, physicians in primary care in Bahrain, whether male or female, are not giving adequate attention to women's sexual health. For these primary care physicians to perform at a higher functional level in the area of women' sexual health, several approaches should be considered. First, there is a need for physicians to learn the communication skills specific to the area of sexual health. Second, all primary care physicians should have access to continuing medical education workshops that focus on the content of sexual medicine and STIs. Third, women physicians involved in reproductive health should have special access to workshops that deal with current clinical practice guidelines. 


\section{References}

1. Report on the global AIDS epidemic 2008. Geneva, Joint United Nations Programme on HIV/AIDS (UNAIDS), 2008.

2. Health statistics of 2008. Manama, Kingdom of Bahrain, Ministry of Health (http://www.moh.gov.bh/PDF/Publications/ Statistics/HS2008/PDF/CH07-publichealth_2008.pdf, accessed 10 May 2012).

3. Cassell JA, Brook MG, Mercer CH. Treating sexually transmitted infections in primary care: a missed opportunity? Sexually Transmitted Infections, 2003, 79:134-6.

4. Richard M. Training for GPs in sexual and reproductive health. British Medical Journal, 2005, 330:225-226.

5. Wiesenfeld $\mathrm{HC}$ et al. Knowledge about sexually transmitted diseases in women among primary care physicians. Sexually Transmitted Diseases, 2005, 32(11):649-653.

6. Cassard SD et al. Physician gender and women's preventive services. Journal of women's health, 1997, 6(2):199-207.

7. Kreuter MW et al. Are patients of women physicians screened more aggressively? A prospective study of physician gender and screening. Journal of General Internal Medicine, 1995, 10(3):119-125.

8. Maheux B et al. Do women physicians do more STD prevention than men? Quebec study of recently trained family physicians. Canadian Family Physician, 1997, 43:1089-1095.
9. Burd ID, Nevadunsky N, Bachmann G. Impact of physician gender on sexual history taking in a multispecialty practice. Journal of Sexual Medicine, 2006, 3:194-200.

10. Lurie $\mathrm{N}$ et al. Why do patients of female physicians have higher rates of breast and cervical cancer screening? Journal of General Internal Medicine, 1997, 12(1):34-43.

11. Wimberly $\mathrm{YH}$ et al. Sexual history-taking among primary care physicians. Journal of the National Medical Association, 2006 98(12): 1924-1929.

12. Tsimtsiou $Z$ et al. Predictors of physicians' involvement in addressing sexual health issues. The Journal of Sexual Medicine, 2006, 3(4):583-588.

13. Khandwalla H, Luby S, Rahman S. Knowledge, attitudes, and practices regarding sexually transmitted infections among general practitioners and medical specialists in Karachi, Pakistan. Sexually Transmitted Infections, 2000, 76:383-785.

14. Gureje $\mathrm{O}$ et al. Somatization in cross-cultural perspective: a WHO study in primary care. American Journal of Psychiatry, 1997, 154:989-995.

15. Aschka $\mathrm{C}$ et al. Sexual problems of male patients in family practice. Journal of Family Practice, 2001, 50(9):773-778.

16. Fathalla MF, Rashad H. Sexual and reproductive health of women. British Medical Journal, 2006, 333(7573):816-817.

\section{Sexual and reproductive health core competencies in primary care: attitudes, knowledge, ethics, human rights, leadership, management, teamwork, community work, education, counselling, clinical settings, service, provision}

Sexual and reproductive health core competencies in primary care sets forth the core sexual and reproductive health (SRH) competencies that are desirable for use in primary health care (PHC). They reflect the attitudes, tasks, knowledge and skills that health personnel in PHC may need, to protect, promote and provide SRH in the community. These competencies serve as the first step for policy-makers, planners, service organizations and academic/training establishments to understand and meet both the education/training requirements and the service-delivery support needed by SRH staff to provide safe, quality SRH care.

The competencies have been developed through a technical consultation of SRH experts in research, education, policy and service, over more than 2 years. The consultation included 2 workshops and many rounds of review in a Delphiresearch-style process. A survey on the role of PHC providers in SRH was also undertaken to inform the competency definitions.

This document is available at: http://whqlibdoc.who.int/publications/2011/9789241501002_eng.pdf 